\title{
Theoretical and Experimental Study on the Influence of External Friction on Inertia Force Propulsion Device
}

\author{
Qingming Wang ${ }^{\mathrm{a}, *}$, Zhixiang Wei ${ }^{\mathrm{b}}$, Jiachen $\mathrm{Ju}^{\mathrm{c}}$ \\ School of mechanical and power engineering, East China University of Science and Technology, \\ Shanghai, China \\ âwangqm@ecust.edu.cn, ${ }^{\text {b } 773520976 @ q q . c o m, ~}{ }^{c} \mathrm{jj}$ c051126@live.cn
}

Keywords: Inertia force propulsion, Lagrange equation, Iterative numerical solution.

Abstract. There are several ways to realize inertia force propulsion. In this paper, we use an eccentric mass block rotating along the circular orbit to achieve, and the structure is symmetrical to counteract lateral movement. We have established the mathematic model by using Lagrange equation, and have got the numerical solution through programming in MATLAB. The result basically consistent with the experimental, it has laid the foundation for further study.

\section{Introduction}

There are a variety of ways to use centrifugal force driving, such as the inertial propulsion, it has the similar principle. Since 1912, there is over 50 patents has been granted to inventors in the inertial propulsion [1]. In this paper, we use an eccentric mass block rotating along the circular orbit to achieve inertia force propulsion, its structure diagram is shown as Fig. 1[2].
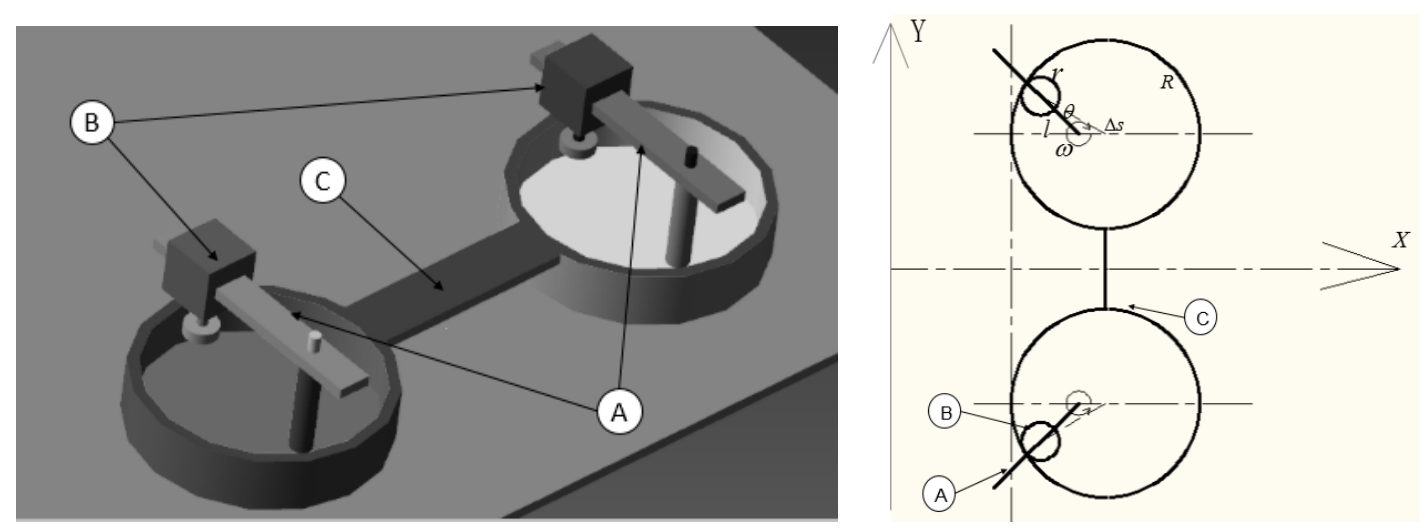

Fig. 1 The structure diagram

In Fig. 1, A, B, C respectively represent the coupler link, the sliding block with truckle and the chassis with circular orbit. The two links A will rotate reversely with the same angular velocity, the sliding block B could slide on the link A, and move along the circular orbit on the chassis $\mathrm{C}$ simultaneously. When the device is running on a surface with friction, it can move along the $\mathrm{X}$ axis direction. The device is the symmetrical structure which has counteracted lateral movement. The specific parameters of our experimental device is as followed, the total weight is $2.01 \mathrm{~kg}$, the weight of a sliding block with truckle is $0.25 \mathrm{~kg}$, the radius of the circle orbit is $6 \mathrm{~cm}$, the eccentric distance is $3 \mathrm{~cm}$, and motor speed is about 126 round per minute. The supports of our experimental device is changeable, so we can get several data under different surfaces. However, because the resource and time is limited, we cannot make too many devices with different size and structure. In order to analyze and optimize the device, it is important to find a mathematic model to realize the numerical simulation and compare it with the experimental data. 


\section{Mathematic Model}

In order to analysis more conveniently, the institution has been simplified, the assumptions are as follow.

This mechanism can be simplified as a flat structure;

All parts of mechanism is rigid. The slider and small wheels are collectively referred to as truckle, and its weight focused on the center of the circle. The weight of the link is regardless, and the weight of the base is uniform distributed;

The coordinate system is in the reference to the ground [3].

The flat structure plane sketch and relevant parameters and symbols are shown as Fig. 1, and $\theta=\omega t, \omega$ is constant. $M$ represent the weight of the chassis, its generalized coordinates are $x_{1}, y_{1}$, $m$ represent the weight of the truckle, its generalized coordinates are $x_{2}, y_{2}$. According to the Lagrange equation [4], the potential energy of the system is constant and the external force is friction force, so we can simplify the equation to the formula (1).

$$
\frac{d}{d t}\left(\frac{\partial T}{\partial \dot{q}_{k}}\right)-\frac{\partial T}{\partial q_{k}}=f
$$

Taking the system as the research object, establish the coordinate system as Fig. 2. Choose $x_{1}$ to be the generate coordinate. Due to the structure's symmetry, we selected as the research object is the truckle located in the top. Then we can get

$$
x_{2}=x_{1}-\Delta s-l \cos \theta, y_{2}=\frac{1}{2} l \sin \theta
$$

Where $l=-\Delta s \cos \theta+\sqrt{R^{2}-\Delta s^{2} \sin ^{2} \theta}$ (Solved by the equation), so kinetic energy of the system is as equal to

$$
T=\frac{1}{2} M \dot{x}_{1}^{2}+\frac{1}{2} 2 m\left(\dot{x}_{2}^{2}+\dot{y}_{2}^{2}\right)=\frac{1}{2}(\mathrm{M}+2 m) \dot{x}_{1}^{2}-2 \dot{x}_{1} m(l \cos \theta)^{\prime}+m\left(\dot{l}^{2}+l^{2} \omega^{2}\right)
$$

Then we can substitute them into Formula (1), one obtains

$$
\ddot{x}_{1}=\frac{2 m_{3}}{m_{1}+2 m_{3}}(l \cos \theta)^{\prime \prime}+\frac{f}{m_{1}+2 m_{3}}
$$

Because $\ddot{x}_{1}$ and $f$ influence each other, we cannot obtain the analytic solution of formula (4), so we try to find the numerical solution through iteration.

\section{Iteration Solution and Friction Model}

According Formula (4), we have got the numerical solution through programming in MATLAB. The progress is, we set the relevant parameters first. In the iterative program, at some point $t_{0}$, to decide the friction force according to the velocity $v_{0}$ first. Then substitute the friction force into formula (3) to get the acceleration $a_{0}$. So we can get the velocity of the next moment which is $v_{0}+a_{0} \Delta t$, and the displacement between the two moments which is $s=s_{0}+v_{0} t$, too. And then to the next moment, repeat the progress until we get the data of the whole progress. The program chart is shown as Fig. 2.

Because iterative numerical solution can only take finite precision, no matter how short the step is, the friction force is discontinuous, and the discontinuity will bring difficulties to the solving process. The velocity and accelerated velocity will oscillate around zero. So we take the approximate model method to avoid this problem, use a straight line of certain slope to denote the friction force around the relative sliding speed zero point. The slope is determined by the velocity limit Dv and the sliding friction force. Then the friction force is a single value function of the relative velocity, the function curve in shown in Fig. 3. It's also one of Karnopp Friction Model [5]. 


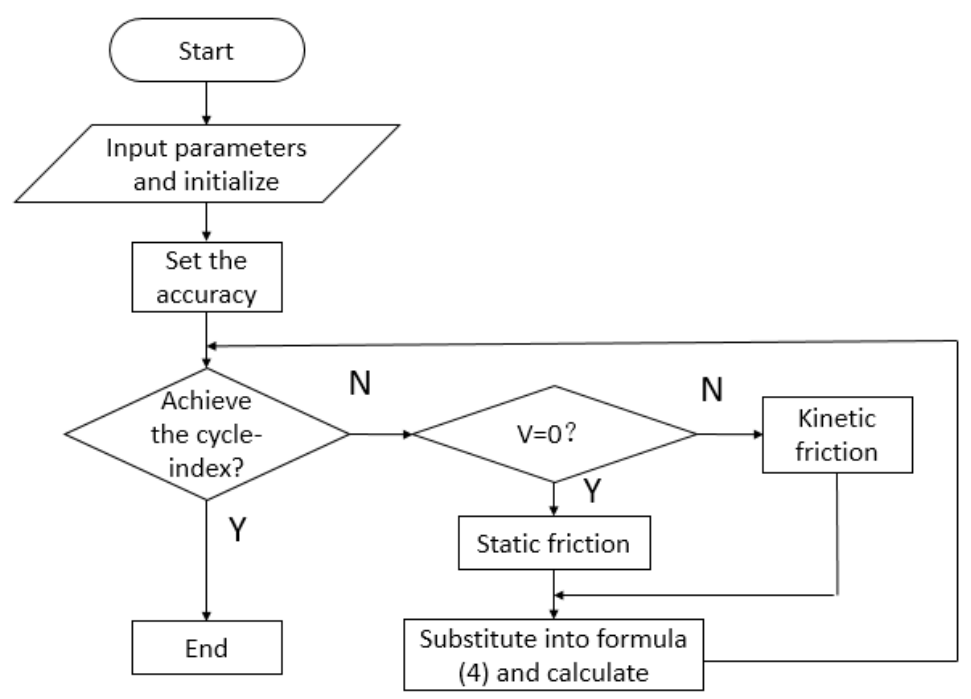

Fig. 2 The iteration solution program chart

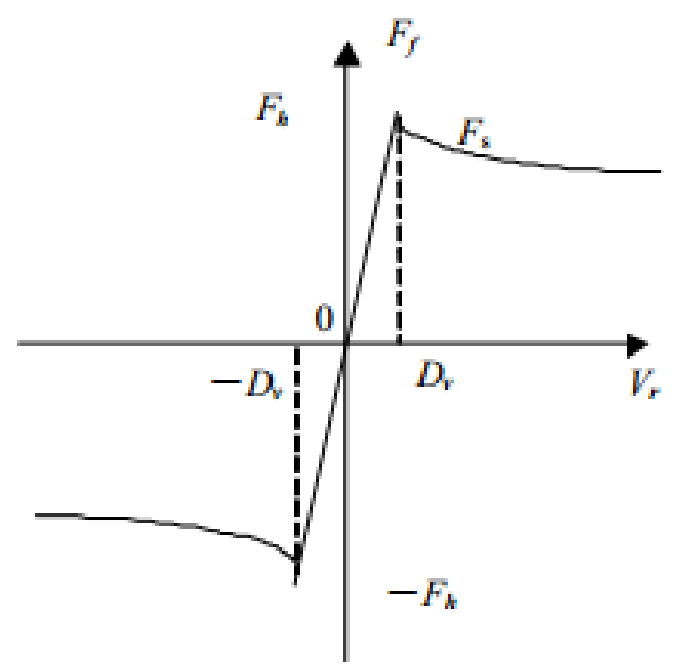

Fig. 3 Friction force approximation function curve

Where $D_{v}$ is the velocity limit, $F_{s}$ is the curve function, we can change them to adjust the accuracy of the result and solve the problem of the oscillation.

\section{Comparison and Analysis}

The results obtained from the parameters in the program as below. In order to verify the iterative numerical solution results, we put the numerical results and the experimental together. The displacement curves are shown in Fig 4, the friction for each figure is static friction force $f_{s}=8.3 \mathrm{~N}$, kinetic friction force $f_{d}=6.9 \mathrm{~N}$ for the left figure, and $f_{s}=7.6 \mathrm{~N}, f_{d}=6.5 \mathrm{~N}$ for the right figure. We use MIRAN KTC-200 mm linear displacement sensor and NI USB 6009 Data Acquisition Card to monitor and record the displacement data, because of the detection precision, there is a noise with the experimental data curve.

As we can see, these two curve are close, both of them can show the progress of moving forward and backward. However, it has a little different of the average speed of the two curve, and the experimental data shows the device move backward more. There are many reasons which could lead to this difference, here is the several possible reasons as listed.

The error of the experimental device such as dimensional accuracy and synchronization;

The error on measurement such as friction coefficient and motor speed;

When the experimental device is running, maybe there are conditions which we haven't taken into account, such as collision and deviating; 
There is still room for improvement on this mathematic model.
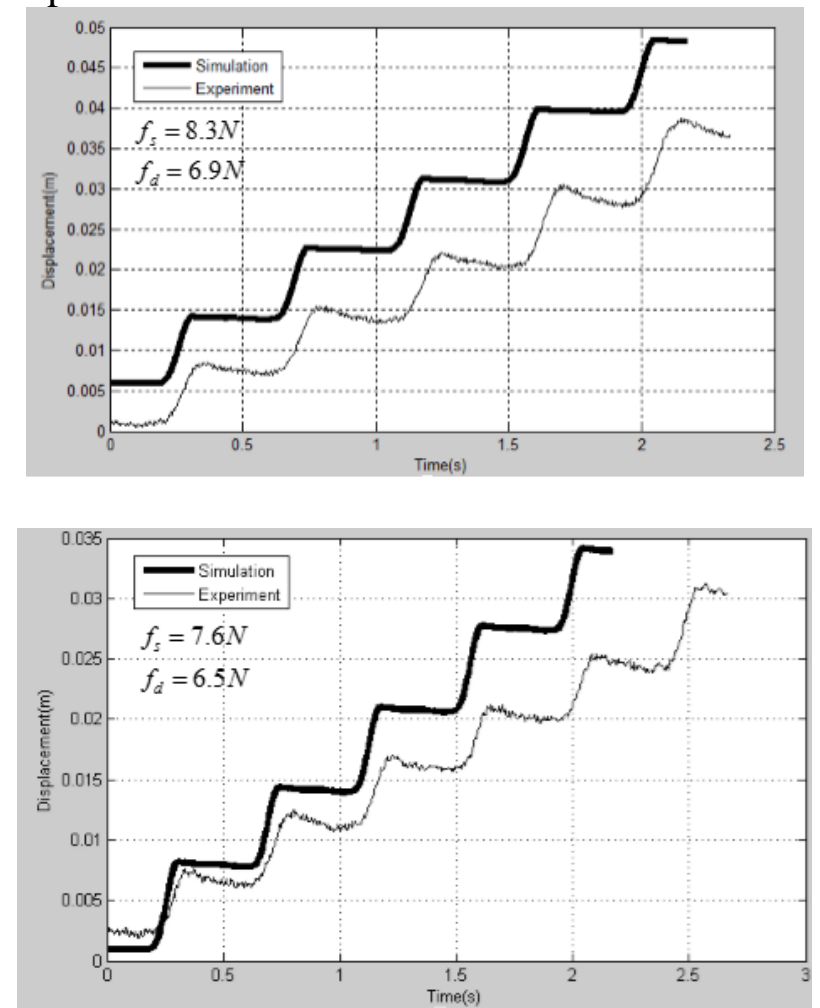

Fig. 4 The displacement curve of numerical and experimental results on different external friction status

\section{Summary}

This paper has established a theoretical model of the inertia force propulsion device, and has used Lagrange equation to derive the dynamic mathematical expression. We use MATLAB to get the results of numerical simulation, the results is identical with the experimental results, and it has laid the foundation for the further study.

\section{Acknowledgments}

This research was supported by National Natural Science Foundation of China (No. 51175176).

\section{References}

[1] Mohanmmed Almesallmy, Experimental and Analytical investigation of Inertial Propulsion Mechanisms and Motion Simulation of Rigid-Multi-Body Mechanical Systems, University of Rhode Island, UMI Microform 3239899. (2006).

[2] Qingming Wang, Jiachen Ju, Analysis of the Effect of External Factors to the Propulsion by Inertial force of Rotating Mechanism, Applied Mechanics and Materials. Vol. 487 (2014) 343-347.

[3] Christopher G. Provatidis, Mechanics of Dean Drive on Frictional Ground, Journal of Mechanical Design and Vibration. Vol. 1, No. 1 (2014) 10-19.

[4] Glocker C, Preiffer F. Complementarity problems in multibody systems planar friction, Archives of Applied Mechanics. 63 (1993) 452-463.

[5] Lilan Liu, Hongzhao Liu, Ziying Wu, Zhongmin Wang, Research Progress on Friction Mode in Mechanical Systems, Advances in Mechanics. Vol. 38, N0.2 (2008) 201-212 